Chrischta Ganz

\section{Die Funktionen der Haut}

Um aufzuzeigen, welch wichtige Aufgaben die Haut übernimmt, sind diese nachfolgend aus naturheilkundlicher Sicht kurz skizziert [1-3].

\section{Schutzfunktion}

Die Haut hat eine grosse Schutz- und Abwehrfunktion und ist gleichzeitig ein wichtiges Immunorgan. Wie eine Barriere schützt die Haut das Körperinnere vor mechanischen, thermischen und chemischen Schäden und wehrt durch ihre exponierte Lage und ihren hohen Anteil an immunologisch kompetenten Zellen Krankheitserreger ab.

\section{Speicherorgan}

Die Haut ist Depot für Wasser, Fett, Blut, Mineralstoffe und Vitamine (bis zu einem Drittel des Blutes kann in der Haut gespeichert werden).

\section{Wärmeregulator}

Durch eine Veränderung der Hautdurchblutung und Flüssigkeitsabgabe über die Schweissdrüsen wird die Körpertemperatur reguliert. Durch normale Hautatmung scheidet die Haut in $24 \mathrm{~h} \mathrm{500-600} \mathrm{ml}$ Wasser aus. Wird zusätzlich geschwitzt, kann die Haut bis 21 Flüssigkeit abgeben.

\section{Sinnesorgan}

Über die Haut nimmt der Mensch Druck, Schmerz, Berührung sowie Wärme und Kälte wahr.

\title{
Hausmittel für die Haut: Vergessene Mittel aus der Hausapotheke und vom Wegesrand
}

Heilpflanzen haben in der Behandlung von Verletzungen und Hauterkrankungen eine lange und erfolgreiche Tradition [1]. Die Haut als grösstes Sinnesorgan (Gesamtfläche 1,5-2 $\mathrm{m}^{2}$, Gesamtgewicht 10-15 kg) reagiert sehr schnell auf äussere und innere Einflüsse, wie viele Redewendungen zeigen: «Das geht mir unter die Haut», «ich muss mich meiner Haut erwehren», «es ist zum aus der Haut fahren», «mir ist nicht wohl in meiner Haut», «mit heiler Haut davonkommen», «dünnhäutig sein» oder «eine dicke Haut haben» und viele andere mehr. Auf der Haut ist die Verbindung zwischen organischen Strukturen und seelischen Vorgängen sichtbar.

\section{Diagnostik und Therapie}

Über die Haut und ihre Erscheinungen können diagnostische Informationen (Krankheiten, aber auch die allgemeine Vitalität) gewonnen werden. Über die reflektorische Verbindung gewisser Hautareale mit inneren Organen (Reflexzonen) kann aber auch direkt therapeutisch Einfluss auf die Krankheitsentwicklung und Genesung genommen werden.

\section{Kommunikationsorgan}

Menschen kommunizieren über die Haut: Wir erröten, erblassen, haben einen Schweissausbruch und senden Sexuallockstoffe aus.

\section{Aufnahmefunktion}

Bekannt ist die Tatsache, dass die Haut Stoffe aufnimmt, z.B. von Hormon- und Nikotinpflastern. Über die Hautatmung wird aber auch Sauerstoff (ca. 4-5\% des $\mathrm{O}_{2}$-Bedarfs) aufgenommen (und Kohlendioxid ausgeschieden).

\section{Ausscheidungsorgan}

Über die Haut wird aber nicht nur Kohlendioxid ausgeschieden, sondern auch viele Stoffwechselendprodukte. In der Naturheilkunde geht man davon aus, dass die Haut neben den Ausscheidungswegen Darm, Leber/Gallentrakt, Nieren/Harnwege, Lymphe und Atmung ein sehr wichtiges Ausscheidungsorgan ist, das über die Talg- und Schweissdrüsen viele Stoffe «entsorgt». Darum wird die Haut auch als zweite Leber oder dritte Niere bezeichnet.

Schwitzen unterstützt durch die vermehrte Ausscheidung über die Haut die Stoffwechselfunktionen und trägt wesentlich zur Entgiftung bei.

Bei vermehrter Stoffwechselbelastung setzt der Organismus in zunehmendem Masse die Haut als Ausscheidungsorgan ein und lagert Substanzen $a b$, die zu Juckreiz, Reizzuständen und Ekzemen der Haut führen können («kompensatorische Ersatzausscheidung»). Durch eine Anregung der anderen Ausscheidungswege wird die Haut entlastet (einer der Gründe, warum die Naturheilkunde die häufigen Cortisongaben in der schulmedizini-

\section{KARGER}

() 2017 S. Karger GmbH, Freiburg

Fax +497614520714 


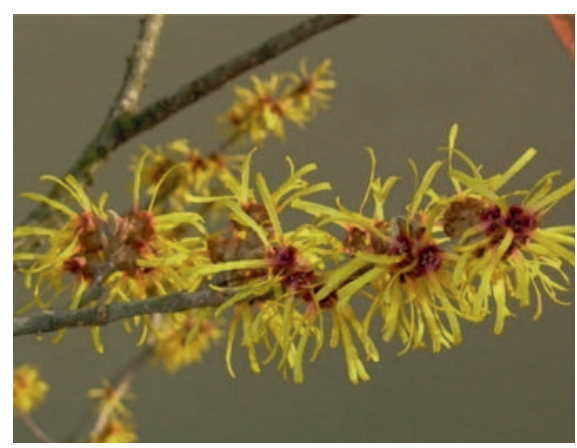

Abb. 1. Die Zaubernuss blüht im Winter.

Kasten 1. Natürliche Zugsalbe

50-70 g Nadelharz und $50 \mathrm{~g}$ feingeschnittene Nadeln werden $2-4 \mathrm{~h}$ bei ca. $60^{\circ} \mathrm{C}$ in $300 \mathrm{ml}$ Olivenoder Rapsöl ausgezogen. In einem separaten Topf wird 30-35 g Bienenwachs geschmolzen und dem Auszugsöl beigefügt. Danach abseihen und unter Rühren abkühlen lassen.

schen Praxis kritisiert). Bei chronischen Hauterkrankungen wird darum nie nur äusserlich behandelt, sondern es werden immer auch stoffwechselanregende Pflanzen eingesetzt - alte, volksheilkundliche Pflanzen wie auch wissenschaftlich gut untersuchte und dokumentierte Heilpflanzen.

\section{Hausmittel und Heilpflanzen für die Haut}

Einige Hausmittel und Heilpflanzen vom Wegrand werden nachfolgend vorgestellt:

\section{Heilpflanzen zur Desinfektion}

[1, 4-6]

Heilpflanzen zur Desinfektion werden als starker Tee (wässriger Auszug) zubereitet, etwas abgekühlt und danach als Waschung oder Umschlag angewendet. Sie wirken entzündungshemmend, granulationsfördernd, antibakteriell, wundreinigend und wundheilungsfördernd. Am bekanntesten sind Kamillenblüten (Matricariae flos), Zaubernussblätter/-rinde (Hamamelidis folium/cortex) (Abb. 1), Ringel-

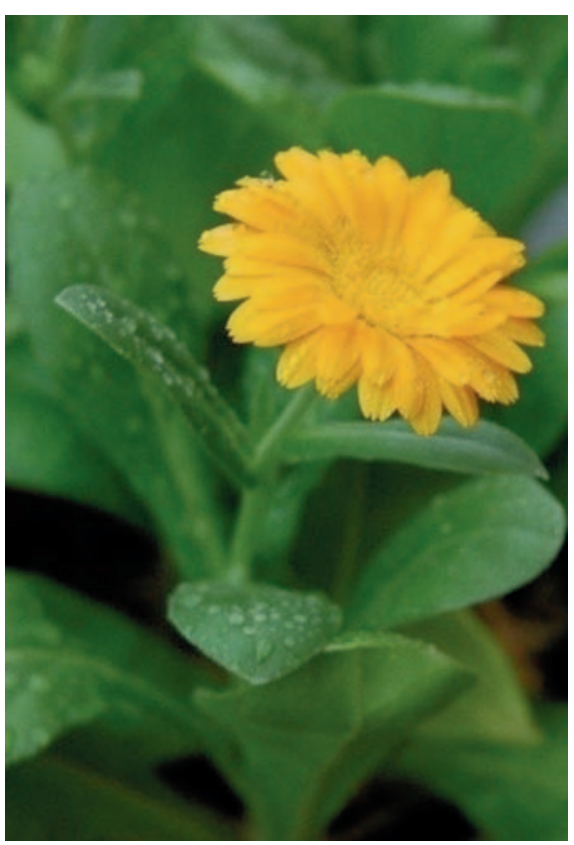

Abb. 2. Die Ringelblume ist eine der bekanntesten Hautpflanzen.

blumenblüten (Calendulae flos) (Abb. 2), Schafgarbenkraut (Millefolii herba), Sonnenhutkraut (Echinaceae herba) (Abb. 3), Sanikelkraut/-wurzel (Saniculae herba/radix), Gänseblümchen (Bellis perennis herba cum flores [7]), Ackerschachtelhalm (Equiseti herba) usw. Auch die meisten einheimischen Ätherisch-Öl-Pflanzen eignen sich zur Wunddesinfektion.

Anwendung: Äusserlich.

\section{Heilpflanzen bei Splitterwunden \\ $[1,4-6]$}

Bei Splitterwunden und verunreinigten Schürfwunden bewähren sich äusserliche Anwendungen in Form einer natürlichen Zugsalbe (bei allen Anwendungen wird danach mit desinfizierenden Heilpflanzen nachbehandelt):

- Eine frische Zwiebelscheibe $1 \mathrm{~h}$ auf die entsprechende Stelle legen; danach lassen sich Splitter einfacher entfernen.

- Als erweichendes Zugpflaster können Bockshornkleesamen- oder Leinsamenmehl als Kompresse aufgelegt werden. Beide Samenmehle wirken erweichend sowie

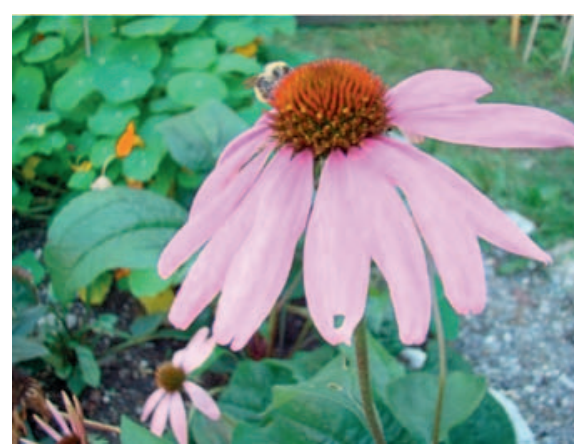

Abb. 3. Sonnenhutblätter können zur Desinfektion von Wunden eingesetzt werden.

entzündungshemmend und ziehen körperfremdes «Material» aus der Wunde.

- Eine natürliche Zugsalbe lässt sich aus dem Harz einheimischer $\mathrm{Na}$ delbäume leicht selber herstellen (Kasten 1).

Heilpflanzen zur Blutstillung [1, 5]

Blutstillende Pflanzen werden erst angewendet, nachdem man die Wunde hat ausbluten lassen, damit die Reinigung der Wunde nicht unterbrochen wird. Ist eine Blutstillung angezeigt, eignen sich Hirtentäschelkraut (Bursae pastoris herba) und Blutwurz (Tormentillae radix) zur äusserlichen Anwendung.

\section{Johannisöl [1, 4-6]}

Weniger bekannt als die innerliche Gabe bei depressiven Verstimmungen ist Johanniskraut (Abb. 4) als äusserliche Anwendung als Ölmazerat, auch Rotöl (Hyperici oleum) genannt. Bereits Paracelsus nannte Hypericum als hervorragendes Wundheilungsmittel; die Wundbehandlung mit Johanniskraut geht jedoch viel weiter zurück. Für die Wundheilung ist vor allem das lipophile Hyperforin verantwortlich. Weitere Inhaltsstoffe sind Hypericine, Flavonoide, Gerbstoffe und wenig ätherisches Öl. Johannisöl wirkt entzündungshemmend, schmerz- und juckreizlindernd, keimwidrig, antibakteriell (unter anderem gegen Staphylokokken), viruzid, antimykotisch und wundheilungsfördernd. Es fördert 


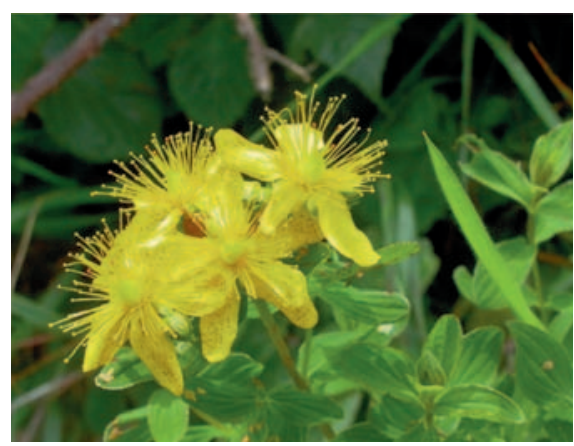

Abb. 4. Das Johanniskraut-Ölmazerat wird in der Behandlung von Wunden sowie bei akuten und chronischen Hauterkrankungen eingesetzt.

die Regeneration der oberen Hautschicht, unterstützt die Hautbarriere und erleichtert eine gute Narbenbildung. Rotöl wird unter anderem eingesetzt zur Behandlung von Schnittund Schürfwunden, Prellungen, Verstauchungen, Verbrennungen ersten Grades, Sonnenbrand, Herpes Zoster, als Dekubitusprophylaxe, zur Narbenbehandlung und bei Amputationsbeschwerden sowie zur Pflege von trockener und rissiger Haut.

Anwendung: Äusserlich.

Sanddorn (Hippophae rhamnoi-

des) $[1,4-6]$

Die korallenroten Beeren des Sanddornstrauches (Hippophae rhamnoides fructus) (Abb. 5) gelten als «Multivitaminbombe» - sie enthalten als einzige Obstfrucht im Fruchtfleisch (nicht im Kern) fettes Öl und damit eine Vielzahl an fettlöslichen Vitaminen. Zur Behandlung von Wunden wird das leuchtend rote und intensiv färbende Fruchtfleischöl verwendet, indem man es pur auf die Wunde aufträgt und mit einer Kompresse abdeckt. Es wirkt entzündungshemmend, antibakteriell, antioxidativ sowie hautpflegend und -regenerierend. Es beugt Pigmentstörungen und der Bildung von Falten vor, schützt die elastischen und kollagenen Bindegewebsfasern, stärkt die Schutz- und Abwehrmechanismen der Haut und bietet einen natürlichen UV-Schutz. Anwendungsgebiete sind Wunden,

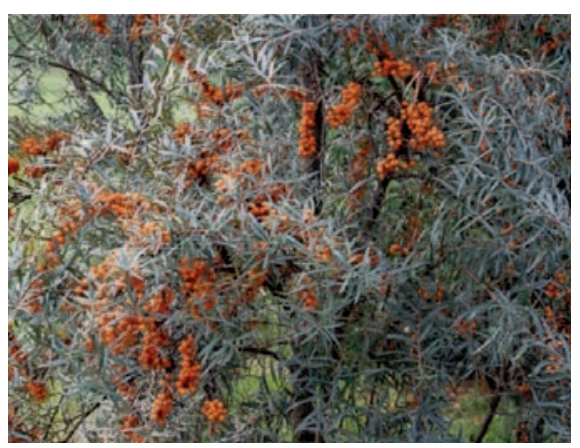

Abb. 5. Aus den leuchtend roten Beeren wird das Fruchtfleischöl gewonnen, das ein effektives Wundheilmittel ist.

Wundliegen, Geschwüre, Ekzeme, schlechte Narbenbildung, Verbrennungen, Sonnenbrand, Sonnenallergie, Erfrierungen und Pigmentstörungen.

Inhaltsstoffe sind Provitamin A (Beta-Carotin), Vitamine B1, B2, B6, B9, B12, C, K und E, gesättigte und ungesättigte Fettsäuren, Flavonoide, Anthocyane, Sterole, Cholin, Mineralstoffe, Fruchtsäuren und Spurenelemente.

Anwendung: Äusserlich (adjuvant innerliche Gabe zur Immunstärkung möglich).

Spitzwegerich (Plantago lanceolata) $[1,4-6]$

Eine der berühmtesten und bewährtesten «Erste-Hilfe-Heilpflanzen» ist der Spitzwegerich, der seit jeher für seine wundheilenden Eigenschaften bekannt ist. Der Saft des Wegerichblattes wirkt reizmildernd, antibakteriell, abschwellend, wundheilungsfördernd, entzündungshemmend sowie schmerz- und juckreizlindernd. Inhaltsstoffe sind Iridoidglykoside (unter anderem Aucubin, Catalpol), Phenylethanoide, Schleimstoffe, Gerbstoffe, Flavonoide, Kieselsäure sowie Kaffee- und Zimtsäurederivate. Zur Wundbehandlung wird ein sauberes Blatt gut gequetscht, bis der Saft austritt, und dieser dann auf die Wunde getropft (oder das gequetschte Blatt als «Wiesenpflaster» auf die betroffene Stelle gelegt). Beim
Trocknen verlieren die Blätter ihre antibakterielle Wirkung (Polymerisierung der Iridoide). Sobald der Saft getrocknet ist, kann das Prozedere wiederholt werden. Auch bei Insektenstichen hat sich diese Methode bewährt. Bei Blasen wird das gequetschte Blatt in den Schuh gelegt oder um die betroffene Zehe gewickelt. Pfarrer Künzle soll auf die Frage, ob bei äusserer Anwendung nicht die Gefahr einer Infektion bestehe, gesagt haben: «Das kennt der Spitzwegerich nicht. Ein solcher Verband ist der erste, manchmal der beste Notverband, denn die Heilung solcher Wunden geht rasch vor sich. Wie mit Goldfäden näht der Wegerichsaft den klaffenden Riss» [1].

Anwendung: Äusserlich.

\section{Walnussbaum (Juglans regia) \\ $[1,4-6]$}

Der Walnussbaum wird vor allem wegen der wertvollen Walnüsse geschätzt. In der Volksheilkunde und der traditionellen europäischen Naturheilkunde (TEN) werden aber auch die Blätter des Walnussbaumes wegen ihrer adstringierenden, entzündungshemmenden, mild oberflächlich anästhesierenden, jurckreizstillenden, sekretionshemmenden, gewebeverdichtenden, kapillarpermeabilitätshemmenden und lymphflussfördernden Eigenschaften eingesetzt. Neueste Forschungsergebnisse zeigten antimykotische und antiseptische Wirkungen (Streptococcus mutans, Staphylococcus aureus, Escherichia coli, Candida albicans). Heute bekannte Inhaltsstoffe sind Gerbstoffe, Flavonoide, Bitterstoffe, Juglon, Phenolcarbonsäuren (Kaffee-, Gallus-, Salicylsäure) und wenig ätherisches Öl. Die wichtigsten Anwendungsgebiete sind chronische Hautentzündungen, Akne, Lidrandund Augenentzündungen, Schleimhautentzündungen, kindliche Dermatosen (Milchschorf, Windeldermatitis) sowie übermässiger Fussschweiss. Gerade bei skrofulösen Kindern sind Walnussblätter das Mittel erster Wahl, da sie (im Gegensatz zu Eichenrinden- 
Anwendungen) die kompensatorische Ersatzausscheidung über die Haut nicht hemmen (Tab. 1). In der TEN werden Walnussblätter auch innerlich in Tee, Tinkturen und spagyrischen Mischungen zur Anregung des Hautstoffwechsels und des Lymphflusses eingesetzt. lich.

Anwendung: Äusserlich und inner-

Nebenwirkungen: Bei längeren äusserlichen Anwendungen Braunverfärbung der Haut.

\section{Kohl (Brassica oleracea) $[1,8]$}

Bei Abszessen, Furunkeln und chronischen Wunden eignet sich eine Kohlauflage. Kohlblätter fördern bei entzündeten oder eitrigen Wunden die Sekretion von Wundsekret nach aussen und reinigen dadurch die betroffene Hautstelle. Für eine Kohlauflage werden frische Kohlblätter von der Mittelrippe befreit, mit dem Wallholz gequetscht, bis der Saft austritt, und aufgelegt. Die betroffene Stelle wird danach bevorzugt mit lauwarmem Kamillentee (statt Wasser) abgewaschen.

Anwendung: Äusserlich.

\section{Hamamelis/Zaubernuss}

(Hamamelis virginiana) [1, 4-6]

Die Zaubernuss (Abb. 1) verdankt ihren Namen nicht nur der Tatsache, dass sie im Winter blüht, sondern auch ihrer zauberhaften Wirkung auf die Haut. Ihre Blätter (Hamamelidis folium) enthalten Gerbstoffe, Proanthocyanidine, Flavonoide, Kaffeesäurederivate sowie ätherisches Öl und

Tab. 1. Der Begriff Skrofulose aus der traditionellen europäischen Naturheilkunde grenzt sich vom (noch) nicht pathologischen Zustand des Lymphatismus ab [2]

\begin{tabular}{ll}
\hline Lymphatismus & «Konstitutionelle Situation, bei der ein humoralmedizinisches Übergewicht der \\
& $\begin{array}{l}\text { kalten und feuchten Qualitäten des Phlegmas vorliegt. Dies zeigt sich in Form von } \\
\text { Krankheiten, die auf Überlastung des Lymphsystems beruhen, beispielsweise Nei- } \\
\text { gung zu wiederkehrenden katarrhalischen Krankheiten, Schwellung von Lymph- } \\
\text { knoten und Mandeln u.v.m.» [2] }\end{array}$ \\
\hline Skrofulose & $\begin{array}{l}\text { "Pathologische Weiterentwicklung des Lymphatismus, bei der das übermässige } \\
\text { Phlegma zusätzlich mit Schärfen verunreinigt ist. Die Skrofulose ist gemeinsame } \\
\text { humoralmedizinische Basis vieler unterschiedlicher Krankheiten und Reaktions- }\end{array}$ \\
&
\end{tabular}

ihre Rinde (Hamamelidis cortex) Gerbstoffe, freie Gallussäure, Ellagitannin sowie wenig Catechingerbstoffe und ätherisches Öl. Blätter und Rinde wirken adstringierend, wundheilungsfördernd, entzündungshemmend, kapillarpermeabilitätshemmend, mild oberflächenanästhesierend, vasokonstriktorisch sowie antioxidativ und verbessern die Hautfeuchtigkeit. Die antioxidativen Flavonoide hemmen die Freisetzung von Histamin und wirken dadurch zusätzlich antiallergisch. Dementsprechend gross ist das Indikationsgebiet der Zaubernuss: Wundheilung bei Hautverletzungen, Abszesse, Sonnenbrand, Verbrennungen, Ulcus cruris, Phlebitis, Dammschnittpflege, Analfissuren, Hämorrhoiden, Entzündungen im Anal- und Genitalbereich, Pflege von trockener Haut (speziell auch Altershaut), akute und chronische Hautentzündungen wie Ekzeme, Neurodermitis, Psoriasis, Juckreiz, Hauterkrankungen im Kindesalter (Milchschorf, Windeldermatitis), Hautpilzerkrankungen.

Anwendung: Innerlich und äusserlich.

\section{Zwiebel (Allium cepa) [1]}

Die Zwiebel wurde bereits im Abschnitt zu den Splitter- und Schürfwunden als Hautmittel erwähnt. Sie zeigt aber auch bei Wespen-, Bienen-, Hornissen- und Hummelstichen eine grossartige Wirkung, wenn kurz nach dem Stich eine Zwiebelhälfte auf das betroffene Hautareal gehalten wird. Die Zwiebel entzieht der Haut das Insektengift, bevor es in den Körper aufgenommen wird, und wirkt starkjuckreiz- und schmerzstillend. Die Anwendung wird nach 10-15 min mit einer neuen Zwiebelhälfte wiederholt.

Anwendung: Äusserlich.

Kartoffel (Solanum tuberosum) [1]

$\mathrm{Zu}$ guter Letzt wird hier noch ein Hausmittel bei verbrannten Fingern vorgestellt. In eine rohe Kartoffel wird ein Loch gemacht und der verbrannte Finger dann längere Zeit in die Kartoffel gehalten. Durch die langsame Verdunstung des Wassers wirkt die Kartoffel kühlend, das Wasser hält die verbrannte Haut feucht und bisher nicht erforschte Stoffe der Kartoffel beschleunigen die Wundheilung.

\section{Literatur}

1 Bühring U: Praxis-Lehrbuch Heilpflanzenkunde. Stuttgart, Haug, 2014.

2 Raimann C, Ganz C, Garvelmann F, BertschiStahl HD, Fehr-Streule R: Grundlagen der traditionellen europäischen Naturheilkunde. Schiedlberg, Bacopa, 2012.

3 Garvelmann F, Raimann C: Humoralmedizinische Praxis. Schiedlberg, Bacopa, 2016.

4 Künzle J: Das grosse Kräuter-Heilbuch. Olten, Otto Walter, 1945.

5 Bühring U: Alles über Heilpflanzen. Stuttgart, Ulmer, 2011.

6 Treben M: Heilkräuter aus dem Garten Gottes. München, Heyne, 1986.
7 Ganz C: Heilpflanze des Jahres 2017: Gänseblümchen (Bellis perennis). Schweiz Z Ganzheitsmed 2017;29:100-102.

8 Thüler M: Wohltuende Wickel. Worb, Maya Thüler, 2003. 